\title{
Una mirada desde la inclusión al Programa de Integración Escolar (PIE) en escuelas rurales chilenas: un análisis de casos
}

A View from the Inclusion to the Scholar Integration Program in Chilean Rural Schools: An Analysis of Cases

Um olhar a partir da inclusão no Programa de Integracao Escolar em escolas rurais chilenas: uma análise de casos

\author{
Carmen Gloria Núñez-Muñoz* iD orcid.org/0000-0001-7252-5031 \\ Mónica Peña-Ochoa** iD orcid.org/0000-0002-1821-0546 \\ Bryan González-Niculcar*** iD orcid.org/0000-0003-0644-0245 \\ Paula Ascorra-Costa**** (iD) orcid.org/0000-0001-9449-8273
}

\footnotetext{
Para citar este artículo: Núñez-Muñoz, C.G., Peña-Ochoa, M., González-Niculcar, B., y Ascorra-Costa, P. (2020). Una mirada desde la inclusión al Programa de Integración Escolar (PIE) en escuelas rurales chilenas: un análisis de casos. Revista Colombiana de Educación, /(79), 347-368. https://doi.org/10.17227/ rce.num79-9725
}

\section{(c) $\underset{\mathrm{Br}}{(\text { i) }(9)}$}

* Doctora en Ciencias de la Educación. Académica de la Pontificia Universidad Católica de Valparaíso, Chile. Correo electrónico: carmen.nunez@pucv.cl

** Doctora en Ciencias de la Educación. Académica Universidad Diego Portales, Santiago, Chile. Correo electrónico: monica.pena@udp.cl

*** Licenciado en Psicología de la Pontificia Universidad Católica de Valparaíso. Profesor Agregado de la Es cuela de Psicología Pontificia Universidad Católica de Valparaíso, Viña del Mar, Chile. Correo electrónico: bryan.gonzalez.n(a)mail.pucv.cl

**** Doctora en Psicología. Docente y directora de la Escuela de Psicología Pontificia Universidad Católica de Valparaíso (PuCV), Viña del Mar, Chile e Investigadora Principal Centro de investigación para la Educación Inclusiva. Correo electrónico: paula.ascorra@pucv.cl 


\section{Resumen}

El Programa de Integración Escolar (PIE) es una política de inclusión escolar que funciona bajo el principio de subvenciones especiales entregadas a niños y niñas con necesidades educativas especiales. Las escuelas rurales también han podido optar a formar parte de PIE. En este artículo se presenta una investigación-acción que buscaba potenciar las prácticas inclusivas de las escuelas rurales. Se levantó información en 6 escuelas rurales de Chile, a través de entrevistas y talleres con miembros de la comunidad escolar. Los resultados destacan tres formas de aproximarse a PIE, dos de ellas de casos de escuelas con PIE y una aproximación que engloba las escuelas sin PIE. Las tres posiciones cuestionan el programa de diferentes formas, mientras que solo en una de ellas el PIE parece ser un programa aceptado y coherente con las prácticas escolares. Se concluye reflexionando sobre el valor de PIE y el problema de la migración de "niños PIE" urbanos a escuelas rurales.

\section{Palabras clave}

escuelas rurales; educación inclusiva; educación rural; política educacional; estudio de casos

\section{Keywords}

rural schools; inclusive education; rural education; educational policy; case studies

\begin{abstract}
The School Inclusion Program (SIP) is a school inclusion policy that operates under the principle of special grants given to children with special educational needs. Rural schools have also been able to choose to be part of sIP. This article presents an action research that sought to promote the inclusive practices of rural schools. Information was collected in 6 schools of Chile, through interviews and workshops with members of the school community. We found three ways to approach sIP, two of them from SIP schools and an approach that encompasses schools without sIP. The three positions question the program in different ways, while only in one of them sIP seems to be an accepted program and coherent with school practices. It concludes by reflecting on the value of sIP and the problem of the migration of urban " sIP children" to
\end{abstract} rural schools

\section{Resumo}

O Programa de Integração Escolar (PIE) é uma política de inclusão escolar que trabalha sob o princípio de subsídios especiais concedidos a meninos e meninas com necessidades educacionais especiais. As escolas rurais também foram elegíveis para fazer parte da PIE. Este artigo apresenta uma pesquisa-ação que buscou promover práticas inclusivas em escolas rurais. As informações foram coletadas em 6 escolas rurais do Chile, por meio de entrevistas e workshops com membros da comunidade escolar. Os resultados destacam três maneiras de abordar O PIE, duas delas de casos de escolas com PIE e uma abordagem que inclui escolas sem PIE. As três posições questionam o programa de maneiras diferentes, enquanto apenas em um deles o PIE parece ser um programa aceito e consistente com as práticas escolares. Conclui refletindo sobre o valor do PIE e o problema da migração de "crianças PIE" urbanas para escolas rurais.

\section{Palavras-chave}

escolas rurais; educação inclusiva; educação rural; política educacional; estudos de caso 


\section{Introducción}

Los análisis que aquí se presentan corresponden a resultados de nuestra investigación "Reconociendo y potenciando prácticas inclusivas en educación rural", realizada entre los años 2016 y 2018 con 6 escuelas básicas rurales de Chile.

Esta indagación es la continuación de un proceso investigativo del equipo acerca de las escuelas rurales en Chile que busca reconocer el lugar y el aporte que cumplen en la educación nacional. Las escuelas rurales se caracterizan por ser espacios educacionales de amplio impacto en las comunidades donde se insertan, afectando no solo a quienes ocupan sus aulas sino también a las familias y habitantes del sector, debido a su importante rol en la cohesión de las comunidades rurales (Núñez, Peña, Cubillos y Solorza, 2016). No obstante su relevancia, de acuerdo con los datos del Ministerio de Educación (s.f.), el año 2000 existían en Chile un total de 3807 escuelas rurales municipales, mientras que en 2018 el total es de 2744, situación que evidencia una clara tendencia a su disminución.

Es relevante señalar que el sistema de financiamiento de las escuelas en Chile, vigente desde 1981, es a través de subvención a la demanda. Este sistema implica que las escuelas reciban fondos económicos a través del número de estudiantes que asiste a sus aulas. Esta lógica implica no solo una perspectiva política y económica específica, donde la descentralización y la competencia toman un lugar preponderante, sino que también es una perspectiva que asume un entorno geográfico particular, que es el urbano de alta densidad donde habría muchos potenciales estudiantes y más escuelas para que los padres elijan. Las escuelas rurales, al estar en espacios geográficos más bien aislados (bajo la suposición de que muchas de ellas se han vuelto peri-urbanas los últimos años con el crecimiento de las ciudades), son parte de espacios muy particulares donde la lógica de competencia tuvo aun menos éxito de lo que ha demostrado tener a nivel urbano (para entender el fracaso del modelo de subvención a la demanda ver McEwan y Carnoy, 2000; McEwan, Urquiola, Vegas, Fernandes y Gallego, 2008).

En otras palabras, esta investigación parte de la base que las escuelas rurales han tenido una relación distintiva con las políticas públicas educativas del país y, aunque están dentro de las lógicas del sistema escolar, operan de manera diferente a lo exigido por este sistema. Por ejemplo, reciben y retienen a todos los niños y niñas que eran parte de otras escuelas rurales y de escuelas urbanas, aun antes de la Ley de Inclusión (2016) que "promueve el principio de no discriminación arbitraria e inclusión y el principio de gratuidad progresiva en los establecimientos subvencionados que reciben aportes permanentes del Estado". Esto no quiere decir que hubiese necesariamente una visión o posición sistemática o cultura inclusiva 
establecida, sino que las escuelas rurales, para su supervivencia, han debido incluir a todos los niños y niñas que pidieran matrícula en la institución, sin generar prácticas de selección inicial o expulsión durante el proceso educativo; ambas medidas ahora prohibidas por la Ley de Inclusión.

Bajo este contexto es necesario analizar las perspectivas que las escuelas rurales tienen acerca del Programa de Integración Escolar (PIE en adelante), ya que la perspectiva de estas es distinta a la de las escuelas para las cuales fueron diseñadas estas políticas públicas. PIE es un programa de subvención especial que buscaba originalmente retener a los estudiantes con necesidades educativas especiales. Su marco legal es el Decreto 170 (2009) y se define como el aumento de la subvención recibida por el niño cuando tiene un diagnóstico que implica dificultades de aprendizaje. Cada escuela postula a través de un proyecto de mejora, en donde se puede financiar infraestructura, así como especialistas para el trabajo con los niños con necesidades educativas especiales (Mineduc, 2017).

En la investigación realizada se definió una muestra que intenta representar la diversidad de escuelas rurales, considerando además aspectos transversales, ya que muchas de ellas tienen altos índices de vulnerabilidad social, pero solo un número reducido de ellas están adscritas al PIE. También es posible observar que, incluso sin ser parte del programa, muchas escuelas a través de sus directivos y profesores tienen una posición sobre la inclusión en la escuela fuertemente relacionada con las definiciones y estrategias del PIE, ya que era común que los directivos y docentes hicieran referencia fundamental para hablar de educación inclusiva a las implicancias del trabajo con estudiantes con dificultades de aprendizaje o con algún tipo de diagnóstico, especialmente de carácter "no permanente" como la ley indica.

Este artículo busca revisar el lugar del PIE en las escuelas rurales con que se trabajó, proponiendo una mirada crítico reflexiva a una política pública que, si bien declara estar orientada a la inclusión, abre múltiples preguntas en relación con este tema. Para ello, se comenzará con un marco acerca de las escuelas rurales en Chile, una descripción acerca del PIE y sus contextos e implicancias, para luego mostrar el diseño metodológico de la investigación y los resultados, discutirlos desde una perspectiva descriptiva y, finalmente, reflexionar sobre el rol del programa en las escuelas rurales, pero también su lugar como operador de la inclusión en sujetos "excluidos" de la educación chilena.

\section{Contexto chileno de las escuelas rurales}

Como en el resto de Latinoamérica, el mundo rural presenta enormes inequidades en relación al medio urbano, así como mucha heterogeneidad (Corvalán, 2006). El medio rural es de mayor pobreza y, al mismo tiempo, 
presenta una gran diversidad cultural, puesto que en él se concentra una mayor proporción de pueblos originarios, lo cual se refleja en la realidad de las escuelas rurales. De este modo, según cifras de la Agencia de la Calidad de Educación, al año 2016 el 91 \% de los establecimientos rurales del país pertenecían al grupo socioeconómico bajo o medio bajo, mientras que en los urbanos esta proporción es del 49 \% (Elige Educar, 2019); por su parte, al 2016, el $26 \%$ de los estudiantes en establecimientos rurales pertenece a algún pueblo indígena, mientras que en los urbanos el promedio es de un $6 \%$ (Elige Educar, 2019).

Asimismo, lo rural en Chile atraviesa por importantes transformaciones en los territorios, de las cuales destaca el crecimiento de las ciudades, que ha estrechado las distancias entre los espacios urbanos y rurales, principalmente en las zonas más pobladas. Las fronteras con lo urbano se han complejizado y se han vuelto más difusas, y a la migración desde el campo a la ciudad que caracterizó la segunda mitad del siglo xx, se suma la migración desde la ciudad al campo en los últimos años (Trimano, 2015). Esto último ha implicado transformaciones en la composición de la matrícula de las escuelas rurales, que no se encuentran sistematizadas, pero que se hicieron evidentes durante la investigación.

\section{PIE, Ley de inclusión y políticas de integración escolar en Chile}

En 2016 entró en vigencia la Ley 20.845, Ilamada "Ley de Inclusión Escolar". Esta prohíbe el lucro de sostenedores privados que reciben subsidio, elimina gradualmente el copago que realizaban las familias en las escuelas subvencionadas por el Estado, pero administradas por privados, y prohíbe la selección de alumnos en todos los niveles de enseñanza que, hasta ese momento, estaba permitida en establecimientos que recibían fondos del Estado. Según Rojas y Armijo (2016), la Ley de Inclusión ha sido un primer paso para generar condiciones administrativas y regulatorias que permitan debilitar, en parte, la dinámica segregadora del mercado educativo chileno.

Antes de esta ley predominaba, dentro de la política educativa en Chile, una perspectiva fuertemente centrada en la integración de las niñas y niños con necesidades educativas especiales (en adelante N.E.E), a pesar de la existencia de herramientas técnicas oficiales, como por ejemplo los "Criterios y orientaciones de flexibilización del curriculum" (Mineduc, 2009), orientadas a la comprensión de la inclusión desde la perspectiva desarrollada por Tony Booth y Mel Ainscow (2000) en su Índice de Inclusión Escolar. Dicho índice se define como "un conjunto de materiales diseñados para apoyar a las escuelas en el proceso de avanzar hacia una educación inclusiva. El objetivo es construir comunidades escolares 
colaborativas que promuevan en todo el alumnado altos niveles de logro" (Booth, Ainscow, Black-Hawkins, Vaughan y Shaw, 2000, p.3). Con esta perspectiva se pone énfasis en que todas y todos los estudiantes desarrollen altos niveles de aprendizaje, sin discriminaciones arbitrarias, se critica el concepto de N.E.E por centrarse en los déficit individuales y darle un rol fundamental a los especialistas, así como diferenciar explícitamente entre N.E.E y discapacidad, aclarando que es posible tener una discapacidad sin que eso signifique una N.E.E.

A partir del año 2009 se comienza a implementar el PIE en Chile. A pesar de su carácter de integración, como lo dice su nombre y también como queda demostrado en sus estrategias de trabajo, este es descrito por la autoridad ministerial como un programa de tipo inclusivo:

El PIE es una estrategia inclusiva del sistema escolar, que tiene el propósito de contribuir al mejoramiento continuo de la calidad de la educación que se imparte en el establecimiento educacional, favoreciendo la presencia en la sala de clases, la participación y el logro de los objetivos de aprendizaje de todos y cada uno de los estudiantes, especialmente de aquellos que presentan necesidades educativas especiales (Mineduc, 2009).

En términos prácticos, el PIE implica fundamentalmente el uso de recursos del Estado en la incorporación de especialistas para el trabajo con estudiantes con N.E.E, de manera que presten "ciertos apoyos de tipo extraordinario durante un tiempo específico o durante toda la etapa escolar dependiendo de la evolución de las necesidades educativas especiales y del mejoramiento de las condiciones del contexto escolar" (Mineduc, 2013). Para el caso del aula multigrado de escuelas rurales, el PIE es propuesto por Mineduc como "una gran oportunidad para las escuelas multigrado" en la medida que permite "brindar una educación pertinente, relevante y significativa a las y los estudiantes con mayor riesgo de fracaso y deserción escolar, que presentan necesidades educativas especiales de carácter permanente y transitorio" (Mineduc, 2013). En el caso de las escuelas rurales, dado el bajo número de estudiantes, entre otras razones, no siempre cuentan con el programa de integración.

El marco legal de PIE es el Decreto 170 (2009), el que establece subvenciones estatales para los sostenedores de las escuelas por cada estudiante que cuente con uno de los diagnósticos contemplados en el decreto. Esto implica que mientras más estudiantes con diagnóstico tenga una escuela, mayor cantidad de dinero recibirá, siempre que no sobrepase el límite de 5 estudiantes con diagnósticos asociados a N.E.E de tipo Transitorio y 2 estudiantes con diagnósticos asociados a N.E.E de tipo Permanente. Según el decreto, se entiende por N.E.E de tipo permanente, aquellas barreras para aprender y participar que determinados estudiantes experimentan 
durante toda su escolaridad como consecuencia de una discapacidad diagnosticada por un profesional competente, y que demandan al sistema educacional la provisión de apoyos y recursos extraordinarios para asegurar el aprendizaje escolar. El decreto refiere los siguientes déficits $O$ discapacidades ${ }^{1}$ de carácter permanente:

» Discapacidad visual

» Discapacidad auditiva

» Disfasia severa

» Trastorno autista

» Deficiencia mental severa o multidéficit

Las N.E.E de tipo transitorias, son aquellas no permanentes que presentan los alumnos en algún momento de su vida escolar a consecuencia de un trastorno o discapacidad diagnosticada por un profesional competente, $y$ que necesitan de ayudas y apoyos extraordinarios para acceder o progresar en el currículum por un determinado periodo de su escolarización. Se incluyen las siguientes condiciones:

» Trastornos específicos del aprendizaje

» Dificultades específicas del aprendizaje de la lectura

»Dificultades específicas de la lectura y escritura

» Dificultad específica del aprendizaje de las matemáticas

» Trastornos específicos del lenguaje (TЕL)

»Trastorno déficit atencional con y sin hiperactividad (TDA) o Trastorno hipercinético

» Rendimiento en pruebas de coeficiente intelectual (CI) en rango límite, con limitaciones significativas en la conducta adaptativa

El año 2013 se publicó la única evaluación de programas PIE existente hasta la fecha. En esta, se consigna al programa como exitoso en términos de implementación y participación, pero aun así se refiere que es un programa que funciona de manera aislada dentro de las escuelas y que no logra ser asociado a conceptos como el mejoramiento y la efectividad escolar (Centro de Innovación en Educación y Fundación Chile, 2013). Este estudio demostró que las escuelas municipalizadas urbanas eran las que tenían mayor participación en PIE. Las escuelas rurales, en cambio, solo representaban un $33 \%$ de las escuelas pertenecientes al programa. El estudio dio cuenta de que el diseño de la política generó problemas de equidad reflejados especialmente en las escuelas rurales, ya que no se tomaron en cuenta variables territoriales como, por el ejemplo, el acceso a profesionales especialistas.

1 Conceptos utilizados en el Decreto referido. 
PIE y su marco legal, el Decreto 170, han sido sujetos de diversas críticas. Peña (2013) considera que el Decreto 170, al concentrarse en el diagnóstico de individuos, solo regula los aspectos diagnósticos para la selección de los estudiantes que recibirán el financiamiento, sin dar indicaciones acerca de los procesos de integración propiamente tales. Rojas y Armijo (2016), ponen el escenario previo a PIE, la estandarización de resultados mediante currículo nacional y pruebas estandarizadas como un contexto adverso para la diversidad en general. Asimismo, señalan que los docentes incorporan dinámicas clasificatorias y estigmatizantes como "niño PIE" O "niño SEP", "intensificando formas de nombrar la diversidad estudiantil desde la exclusión" (Rojas y Armijo, 2016, p. 10). En el estudio de Tamayo, Carvallo, Sánchez, Besoaín y Rebolledo (2018), orientado a evaluar PIE en el área de discapacidades motrices y sensoriales (las que por ley, en el caso chileno, son llamadas "permanentes"), se considera que los proyectos del programa de integración están orientados a la contratación de profesionales psicólogos, quienes son un personal hasta ahora no capacitado para trabajar con estudiantes que presentan este tipo de necesidades educativas. Asimismo, se indica que todavía hay baja accesibilidad y participación de estudiantes y comunidades en general en las escuelas que Ilevan a cabo proyectos de inclusión.

\section{Metodología}

Se realizó una investigación cualitativa con base en los fundamentos y técnicas de la etnografía clásica y la cuasi etnografía (Silva y Burgos, 2011; Iñiguez, 2004). En ella se incorporaron, además, los supuestos de la investigación acción (Balcázar, 2003; Carr y Kemmis, 1988; Elliot, 2005); esto con el objetivo de reconocer y potenciar prácticas, políticas y culturas inclusivas en seis escuelas rurales de Chile. Desde esta perspectiva, la comunidad educativa, principalmente sus docentes junto a una dupla de investigadores, quienes a su vez cumplieron un rol de par crítico, formaron parte de la producción de conocimiento situado que se constituyó como la base para el diseño e implementación de acciones conducentes a fortalecer las culturas inclusivas de las escuelas participantes.

A continuación, se describen en términos generales las fases estructurantes del proceso investigativo:

a. Fase de campo: producción de información bajo la perspectiva cuasi etnográfica. En ella se incluyen distintos actores de la comunidad educativa, bajo la consideración que se espera generar un conocimiento desde y con sus involucrados. Asimismo, se tienen en cuenta diversos espacios de la cotidianeidad de la escuela. 
b. Fase de análisis: análisis de la información producida. En este estudio se incluyen dos niveles: el dependiente del equipo de investigadores y un nivel considerado como análisis conjunto (con actores de las comunidades educativas) de la información. Esto como fundamento para asegurar la factibilidad y concreción de la fase siguiente.

c. Fase de elaboración de planes de acción: esta fase corresponde a un proceso de acompañamiento y asesoramiento a la comunidad escolar en la elaboración participativa de planes de acción que potencien las prácticas inclusivas, así como también para la creación de estas en virtud de las necesidades particulares y recursos de cada escuela. En esta fase, el rol de los investigadores se constituye como un amigo crítico externo a la escuela y como un agente facilitador de la sistematización de las acciones desarrolladas. En relación a esto último, se incluyen acciones de trabajo con microcentros (instancia pedagógica de reunión mensual de escuelas rurales próximas en términos geográficos), basada en acciones de transferencia y reflexión en torno a las culturas, políticas y prácticas de inclusión con las escuelas, y un encuentro final de sistematización de las acciones realizadas en el que participaron representantes de las seis escuelas en conjunto con las y los investigadores del proyecto.

Las escuelas participantes fueron escogidas a partir de 5 criterios preestablecidos y orientados a incorporar una mayor diversidad de escuelas, en consideración del modelo metodológico y de los alcances del estudio. No obstante, la participación de las escuelas se concretó mediante un diálogo para la generación de acuerdos y enunciación conjunta de los sentidos de participación.

Los criterios incluyeron distinciones en 1) el tipo de estrategias de enseñanza, es decir, escuelas multigrado o de curso combinado unidocente o no unidocente; 2) el Índice de Vulnerabilidad Escolar (IVE-SINAE) que determina, mediante diversas variables, un porcentaje de estudiantes vulnerables por establecimiento; 3) el tipo de proyecto educativo, diferenciando aquellos en que se establece explícitamente un proyecto educativo intercultural de las que no; 4) las N.E.E que se traduce en si el establecimiento cuenta con PIE. Si bien comprendemos la inclusión escolar como un proceso que va más allá de la integración de niños y niñas con N.E.E al sistema educativo, consideramos este criterio como relevante en la medida en que ha sido incluido como posibilidad para la escuela rural. Y 5) la ubicación en el territorio nacional, en que se diferenciaron tres categorías generales: norte, centro y sur con el fin de incluir distintas condiciones contextuales. 
De acuerdo con lo anterior, las características de las escuelas participantes se pueden comprender en la tabla 1:

Tabla 1.

Caracterización de escuelas participantes de acuerdo a criterios de selección

\begin{tabular}{llllll}
\hline Escuela & Criterio 1 & Criterio 2 & Criterio 3 & Criterio 4 & Criterio 5 \\
\hline Escuela 1 & $\begin{array}{l}\text { Multigrado no } \\
\text { unidocente }\end{array}$ & IVE: 76\% & - & C/ N.E.E & Norte \\
\hline Escuela 2 & $\begin{array}{l}\text { Multigrado no } \\
\text { unidocente }\end{array}$ & IVE: 81\% & Intercultural & C/ N.E.E & Sur \\
\hline Escuela 3 & $\begin{array}{l}\text { Multigrado } \\
\text { unidocente }\end{array}$ & IVE: 100\% & - & C/ N.E.E & Centro \\
\hline Escuela 4 & $\begin{array}{l}\text { Multigrado no } \\
\text { unidocente }\end{array}$ & IVE: 91\% & Intercultural & C/ N.E.E & Norte \\
\hline Escuela 5 & $\begin{array}{l}\text { Multigrado no } \\
\text { unidocente }\end{array}$ & IVE: 91\% & Intercultural & C/ N.E.E & Centro \\
\hline Escuela 6 & $\begin{array}{l}\text { Multigrado no } \\
\text { unidocente }\end{array}$ & IVE: 100\% & & S/ N.E.E & Sur \\
\hline
\end{tabular}

Fuente: elaboración propia

En el caso de este trabajo en particular, se utilizó como método el análisis de contenido temático. Proceso que, de acuerdo con Vázquez (1994), consta de tres etapas consecutivas y recursivas: 1) Etapa de preanálisis, que implica la revisión repetida y sistemática de toda la información producida armando a partir de esto el corpus de información para el análisis; 2) etapa de codificación o fragmentación del texto, en la que se establecen las unidades de registro y de contexto, esta etapa incluye la codificación abierta y la codificación axial (Corbin y Strauss, 2008); y 3) Etapa de Categorización, la cual se basa en la agrupación de las unidades en virtud de la temática de estudio y en función del criterio semántico (en términos de similitud de significados) establecido en la etapa anterior.

En consonancia con lo anterior, se organizaron los resultados en torno a las temáticas PIE de una manera muy cercana a un caso de estudio; focalizándose en 3 de las 6 escuelas participantes, que, si bien no era la propuesta original, se relaciona con la fuerte condición de singularidad de cada uno de estos establecimientos, de sus experiencias con el tema, los factores relacionados a la territorialidad y cercanía a las ciudades.

En términos éticos es importante destacar que la participación de los involucrados en esta investigación fue de carácter voluntaria y acordada mediante consentimiento informado individual. 


\section{Análisis y discusión de los resultados}

\section{Escuela 3: el PIE como lavado de conciencia}

Esta es una pequeña escuela de un área agrícola en la zona central del país. En los últimos años ha tenido diversas amenazas de cierre, lo que sin duda ha generado una posición particular de sus profesionales y, en especial, respecto de la necesidad de atraer matrícula para evitar el cierre. Esta situación, sabemos, genera fuertes problemas dentro de las escuelas, la administración y las comunidades (Solís y Núñez, 2014; Núñez, Solís y Soto, 2014; Vera-Bachman y Salvo, 2016).

EI PIE en esta escuela está conformado por 4 profesionales: una psicóloga, una psicopedagoga, una profesora de aula y una terapeuta ocupacional. Como en el resto de las escuelas rurales con el programa de integración, se trata de profesionales que rotan por horas en las escuelas PIE de la zona. En el caso de la escuela 3, el director describe que el trabajo de las profesionales está poco integrado con el resto de la escuela, con profesionales que se ven poco entre ellas, que él mismo pocas veces alcanza a ver, incluso a saludar o despedirse.

El caso de la escuela 3 refleja una realidad cada vez más común en las escuelas rurales peri-urbanas. Son escuelas que reciben muchos niños que han salido de las escuelas urbanas, aún con "Ley de inclusión" que no permite expulsiones, pero sí como niños "con N.E.E" cuyas familias buscan, de manera supuestamente voluntaria, escuelas más acordes a estas necesidades. La escuela 3 tiene una matrícula de un $100 \%$ de niños provenientes de escuelas urbanas. Recordemos que esta escuela está en riesgo de cierre, amenaza que viven hace varios años. Al respecto, cuando las investigadoras le preguntan la opinión a la psicóloga (parte de la dupla psicosocial de la escuela), ella dice no haberse dado cuenta del problema, pero les encuentra razón a las investigadoras. Su explicación es que los padres de niños con N.E.E buscan escuelas "pequeñas" que brinden atención personalizada a los niños. El uso de escuela "pequeña" para referirse a las rurales y escuela "grande" a las urbanas se repite en otras escuelas investigadas y se considera, en general, una característica positiva de las escuelas rurales. Tanto el director de esta escuela como el profesor encargado de la escuela 2, dijeron que uno de los temas importantes para ellos es integrar a los niños de las escuelas grandes, que son vistas por ellos y sus comunidades como violentas y a la vez indiferentes con los niños.

Esa indiferencia es a la que se refiere la psicopedagoga:

De que el encontrarse en un sistema educativo de una escuela por ejemplo de mil alumnos, ¿cierto? Que su hijo tenga ciertas necesidades educativas especiales o que tenga ciertas dificultades, a lo mejor 
ese apoderado o ese alumno no se va a sentir del todo apoyado o resguardado. Entonces van a buscar un lugar que sí se lo pueda dar de forma más centralizada, que se enfoque más en algo (sic) (Psicopedagoga escuela 3).

En este caso, la atención personalizada de los estudiantes parece ser fundamental para la superación de sus problemas de aprendizaje, mucho más que el propio programa de integración pie. Según la psicóloga, trabajan de manera individual y fuera de la sala con cada alumno que está "descendido" según las mediciones que ellas hacen, pero que también hace el programa "Mentor" contratado por la comuna. Trabajar lo individual desde PIE, pero también desde las lógicas que impone la convivencia escolar, se hacen evidentes para la profesional:

Pero yo creo que va a llegar un punto en que ya no, el trabajo individual que nosotros [...] lo que nosotros estamos pudiendo hacer acá es trabajar la complejidad desde lo individual. Entonces yo creo que ese es el paso uno que se da. Que era un poco lo que venía haciendo del año pasado [...], de convivencia podemos hacer va a tener un tope, o sea no vamos a estar con los niños todo el año trabajando ¿cierto? Porque no lo necesitan tampoco, no todos por lo menos. Yo creo que de los técnicos que he tomado, quizás solo uno o dos necesitan apoyo en términos de convivencia escolar, y no es el rest (sic) (Psicóloga escuela 3).

La complejidad de lo dicho refleja muy bien el problema de un PIE poco conectado con el proyecto educativo. Se mezcla con el tema de convivencia que, por lo general, es otra exigencia del sistema escolar a los psicólogos. ¿Cómo el trabajo individual de PIE produce efectos a nivel aula? La convivencia es entendida también por la psicóloga de la escuela 3 como un problema individual como otro déficit que la profesional debe sanar o mejorar para devolver a la sala niños que puedan "convivir". No todos los niños con "déficit" de convivencia están en el programa de integración. La lógica de la explicación es un poco confusa en el texto, pero es el efecto de haber llevado al extremo la lógica del déficit individual trabajado fuera del aula, que se encuentra con las múltiples exigencias que el sistema escolar hace en relación a los déficits de los niños: déficit a nivel de aprendizaje, y déficit a nivel de convivencia, corren por caminos separados.

¿Funciona PIE en esta escuela? ¿Tiene efectos sobre los niños que son parte del programa? La visión de la escuela hacia el programna de integración es altamente crítica. La crítica que hacen podría caracterizarse en tres niveles: Un nivel general como crítica a la política de Estado que no se hace cargo de los verdaderos problemas a la base de la exclusión. Al respecto, el director de la escuela 3 considera que PIE "es un lavado de conciencia del Estado". Repite esto varias veces, en distintas entrevistas 
y encuentros. No obstante, su crítica no es a la segregación que podría generar el diagnóstico, sino que pasa a otro nivel, un segundo nivel que se relaciona con las prácticas que PIE generaría: prácticas altamente burocráticas, un incesante "llenado de papeles" que sería la principal labor de las profesionales. En 2013 esto apareció en la evaluación del programa, que demuestra que el Mineduc se transforma en un evaluador de él, centrado en el control y la evaluación, sin entregar apoyo técnico a las escuelas (Centro de innovación en educación y Fundación Chile, 2013).

Finalmente, hay un nivel de crítica al trabajo de las psicólogas, a quiénes considera jóvenes e inexpertas, que no saben "muñequear" con los niños. Es decir, que se dejarían manipular por estos, ya que describe a los niños como seres que manipulan y que deben ser manejados con férreo control:

Entonces, generacionalmente, con las profesionales PIE tenemos hartos años de diferencia, entonces las chiquillas, hay tratos que a lo mejor no les gusta como soy con los niños. Por ejemplo, yo me coloco en el almuerzo y les hago comer toda la comida (sic) (Director escuela 3).

Otro elemento crítico menos claro, pero que amerita ser analizado, es lo que comenta una de las profesoras de esta escuela. En reunión con representantes de otras escuelas en la instancia de microcentro, relata que la escuela 3 pasó de tener niños "motivados por el aprendizaje" a "un nuevo tipo de matrícula" (que correspondería a los niños que vienen de escuelas peri-urbanas que no tendrían esa motivación por aprender). Asimismo, que uno de estos niños de esta nueva etapa, pasó de ser un niño "terrible", descrito como desmotivado y con déficit atencional, que pasó por el programa —en el sentido que fue "un niño PIE" — a ser un niño motivado y exitoso escolarmente. Su relato da cuenta de que ese cambio es fruto del trabajo docente, mientras que PIE aparece más como un factor para describir un niño problemático que un factor de mejora en ese niño.

Es importante señalar que el plan de acción que esta escuela decidió llevar a cabo consistió en articular la diversidad de programas de intervención que operaban en ella, de modo que hicieran sinergia para desarrollar mejores prácticas inclusivas disminuyendo así la sobreintervención. Por ejemplo, existía el caso de un estudiante que era tratado por tres psicólogos simultáneamente que pertenecían a distintos programas, entre ellos PIE.

¿Ayuda pIE a los niños de esta escuela? No hay elementos que nos permitan asumir que esto sea así, pero tampoco que no. Los tiempos cronológicos de la escuela, la escasa información sobre la labor profesional que realizan las profesionales del programa y la multiplicidad de variables involucradas, hacen de la evaluación del valor de él en la escuela un hecho casi imposible y que es necesario pensar. 


\section{Escuela 4: el PIE como desafío de trabajo curricular}

Esta escuela multigrado se declara intercultural y cuenta con tres profesoras en ejercicio propone una mirada distinta del PIE. De acuerdo con lo declarado, su proyecto educativo se basa en la revitalización de la cultura de los pueblos originarios en Chile. Este proyecto integra distintos aspectos de la cosmovisión de las culturas originarias tales como lengua, gastronomía, celebraciones y la relación con la naturaleza a partir de actividades integradas al currículum y sostenidas institucionalmente en el Programa de Educación Intercultural Bilingüe y el Plan de Acción Ambiental. Parte de su misión es "Trabajar pedagógica y comunitariamente para favorecer el desarrollo integral de los estudiantes en el ámbito intelectual, afectivo, físico, cultural, social y espiritual. A través, de experiencias significativas de participación" (Manual de Convivencia Escuela 4, p. 4).

Con PIE el trabajo realizado está muy teñido por la pregunta de la inclusión en el aula desde hace varios años, que las lleva a cuestionar el modelo de sacar a los niños del aula — forma tradicional del trabajo PIE-, promoviendo, por el contrario, una mirada donde lo curricular y el aprendizaje toman un lugar preponderante: "[...] sacarlos de la sala a enseñarles a multiplicar, a enseñarles a dividir aparte, y que se pierdan el contenido, que es el contenido de la clase de su nivel, no sé si será tan bueno" (sic) (Docente escuela 4). De esa manera, describen el trabajo como un trabajo conjunto, donde el programa de inclusión queda subordinado al trabajo curricular y de aula:

[...] ella [la profesional PIE] va a la sala. Pero cuando tiene que evaluar, ¿cierto? En forma más personalizada, ahí saca al niño o a la niña, pero ella hace el trabajo en aula. Entonces, por ejemplo, yo hago la clase para todos igual, pregunto a todos, PIE no PIE, da lo mismo, pero todos tienen que participar. Entonces después cuando vamos al trabajo práctico, ella se sienta con la persona y le empieza a preguntar o a ayudar, cosa que sea más fácil la internalización del contenido (sic) (Docente escuela 4).

En el escenario anterior, la participación de las y los estudiantes se torna un valor fundamental del trabajo del aula y del aprendizaje. Se da cuenta también de ciertos niveles de coordinación entre las profesionales. Dentro de esta coordinación es que aparece el "Decreto 83" (2015) sobre adaptación curricular que define "distintas formas de aprendizaje" es discutido y aplicado por las docentes:

Yo trabajo harto con [otra docente] en el programa. Articulamos y todo el tema, y también veo, por ejemplo, si voy a pasar un contenido trato de buscar hartas formas de que ellos aprendan, por ejemplo, con videos, con disertaciones, con power [point], con guías, con libros, con hartas formas. Porque tú sabes, los niños aprenden de diferentes maneras. Hay 
unos que les cuesta más, otros menos. Otros tienen más habilidades visuales, auditivas, entonces mi forma de enseñar es de todo un poco y al evaluar también. De repente evalúo informes escritos, disertaciones, pruebas, cuestionarios, guías (sic) (Docente escuela 4).

Las profesoras de esta escuela se muestran en las entrevistas con las investigadoras, cohesionadas, con mucha claridad en relación con los objetivos que buscan lograr. Sus críticas a PIE son de carácter curricular, asumiendo que, si las profesionales entran al aula, tienden a concentrarse en algunas asignaturas más que otras:

P1: Yo encuentro que ellos van, por ejemplo, al aula, a lenguaje, matemáticas no más y dejan de lado ciencias naturales, historia. Que igual son dos ramos fuertes. Ahí yo encuentro que no... que deberían ser los cuatro...

F2: Como focalizado en estos dos que son los, las asignaturas...

P1: Claro.

F2: A las que más se le pone énfasis.

P2: Es que siempre las, lenguaje y matemáticas están en la mira, siempre es como, bueno no solamente PIE, vienen las... los, en este caso... bueno este último año que vino, pero... Chile también siempre era lenguaje y matemáticas (sic) (Taller Docentes escuela 4).

De esta manera, los profesionales del programa PIE, junto a las docentes, han avanzado en un proceso de articulación para incluir lo que refieren como los "distintos estilos de aprendizajes" de sus estudiantes. Esta articulación se da principalmente en las asignaturas de lenguaje y matemáticas, lo que se debe a que el sistema de medición de la calidad de la educación (Simce) se focaliza en dichos subsectores. Esto pone en definitiva sobre la mesa un nivel más amplio respecto de la inclusión: la articulación entre profesionales se basa en cómo adaptar las didácticas y evaluaciones, pero los contenidos no son modificables.

El plan de acción de esta escuela se centró en el trabajo con las familias, con el ánimo de avanzar hacia una comunidad más inclusiva. Uno de los ámbitos considerados fue la reflexión en torno al sentido y uso de los diagnósticos con el fin de evitar prácticas de etiquetaje hacia las niñas y niños que pertenecen a PIE y a sus padres, madres y tutores.

\section{Escuelas sin PIE: el PIE como forma de exclusión propiamente urbana}

Durante el proceso investigativo fue común encontrarse con posturas críticas respecto a las escuelas urbanas y, en general, de los entornos urbanos. La escuela urbana aparece, en comparación con la escuela rural como un 
opuesto: grande, impersonal, desapegada de los estudiantes y/o violenta. En comparación, la escuela rural aparece como pequeña y fuertemente orientada a los y las estudiantes. Esta matriz de análisis aparece también cuando se habla de PIE.

Si estuviéramos en lo urbano ya estarían todos etiquetados. Los niños acá son totalmente diferentes a lo urbano. En la escuela urbana saben qué niños son problemáticos porque van al PIE, al psicólogo o a la asistente social, aquí no pasa eso. El ser rural nos deja con menos recursos y por eso aquí no hay psicólogo o asistente y eso es mejor. Se trabaja con los que tienen que trabajar y es un buen sistema. Una fortaleza de inclusión es que aquí solo estamos nosotros (sic) (Docente en taller, escuela 6).

"Una fortaleza de la inclusión es que aquí solo estamos nosotros". Esta escuela es una escuela rural peri-urbana, a pocos kilómetros de la ciudad principal, capital provincial, con transporte público que permite un fácil acceso. Es una escuela multigrado con una infraestructura muy parecida a cualquier escuela urbana: varias salas, dos patios, espacio para los profesores. No obstante, no tiene PIE, aunque reconocen que hay estudiantes que "vienen" con diagnósticos desde los centros educacionales urbanos. Así, el diagnóstico y lo urbano toman un lugar casi indiferenciable.

Lo mismo pasa en una escuela multigrado unidocente en la misma zona. El diagnóstico es un dispositivo que viene con los niños desde la escuela urbana y desde los programas de integración. Al no haber continuidad de tratamiento dentro de la escuela en las escuelas rurales sin PIE el diagnóstico queda en un lugar externo. Esto generó en ambos casos interesantes conversaciones en relación a los sujetos diagnosticados (ambos casos varones de entre 8 y 10 años con diagnóstico de trastorno de espectro autista). Ambas conversaciones dan cuenta de los comportamientos de los niños, de los signos que estos presentan que inmediatamente se interpretan como síntomas. Acá se describen las formas de habla de estos niños, las dificultades con los profesores/adultos y, fundamentalmente, las dificultades de trato con sus pares en la sala de clases. Asimismo, las estrategias de aula que los profesores y profesoras describen que usan para trabajar con estos niños se salen de las lógicas diagnóstico-psiquiátricas de PIE y generan otro tipo de diálogo. En el caso de la escuela multigrado y unidocente las estrategias son, de fondo, mantener a los niños funcionando dentro del aula con los pares. El que no existan otros espacios obliga a pensar estrategias inclusivas de aula donde, por ejemplo, la salida de la sala es a conversar con el profesor sobre su comportamiento, pero no se usa (porque es difícil de gestionar) la salida de la sala como gestión disciplinar. 
Es importante agregar que la presencia del PIE en las regiones donde se encuentran las escuelas es una de las más bajas a nivel nacional. Cuenta con 25,9 \% y 34,3\% de escuelas en el programa (Centro de innovación en educación y Fundación Chile, 2013).

Los recursos propios para generar una sala inclusiva se desarrollan por fuera de las lógicas diagnósticas y en el caso de la escuela 6, altamente crítica con PIE, es posible vislumbrar una posición contraria hacia el etiquetamiento de los estudiantes y la imposición de formas foráneas de diagnóstico. Además de esto, tienen fuertes aprehensiones hacia la integración entre profesionales de la escuela: la dificultad de hacer un trabajo coordinado con psicólogos y otros profesionales aparece como un desafío que generaría sobrecarga en los profesionales de la escuela. Ahora bien, esta posición crítica podría estar mezclada con el miedo a lo nuevo como una forma de protección a ciertas prácticas conocidas y que debe ser tomada en cuenta cuando se analiza la posición de lo rural en relación con un contexto que suele ser amenazante, en la medida en que la escuela rural es un tránsito a lo urbano en la mayoría de los casos, y lo urbano es la medida hegemónica de los procesos rurales en la lógica de las políticas educacionales chilenas.

Ahora bien, no existe una valoración uniforme con respecto al PIE por parte de los docentes, pues hay posiciones distintas con respecto a él. Algunos reconocen en el PIE un recurso importante para atender a la diversidad de las y los estudiantes. Conviene aclarar que debido al bajo número de estudiantes matriculados, no todas las escuelas rurales cuentan con él. Frente a ello, lo común suele ser que las escuelas reclamen su derecho a tenerlo. Sin embargo, tal como vemos en esta escuela, la incorporación de especialistas es vista como una amenaza a la cultura inclusiva construida, más que un apoyo para consolidarla.

El sacar a los niños de la sala vuelve a ser uno de los problemas de PIE, dado que al hacerlo no hay inclusión sino exclusión:

[...] entonces para mí la inclusión es tomar esos niños y tratarlos como el común de los otros, o sea, nivelarlos para arriba porque para mí. O sea, mi opinión, mi forma de ver el tema es que se nivelan para abajo, de la otra forma, porque tú los sacas de su nivel lo llevas lo trabajas a parte y se perdió todo lo otro (sic) (Docente escuela 6).

PIE produciría cierto tipo de subjetividad de los niños participantes infantilizada, niños que pierden autonomía y motivación por el trabajo. La ayuda que brindaría el programa de integración es leída más como una pérdida de independencia que como una forma de inclusión de sujetos autónomos, lo que nos hace pensar de nuevo: ¿qué resultados genera PIE en los niños y niñas? 
No he trabajado en escuela que lo tiene, pero he recibido a niños que vienen de allá y la mamá llega acá y nos entrega al niño y nos dice: “¿sabe qué, tía? Él viene, él estaba en el PIE, estaba con psicólogo, estaba con esto, estaba con lo otro". Llegan, dejan a sus hijos y les dicen "trabaja como puedas, no te esfuerces, no te canses" (sic) (Docente escuela 6).

En síntesis, creemos que los resultados de la investigación nos dejan varios elementos importantes a tomar en cuenta para comprender PIE en las escuelas rurales y urbanas:

» PIE puede tomar las características de los Proyectos Educativos Institucionales de cada establecimiento educacional si estos son fuertes y comandan también el trabajo dentro del aula.

» Se valora de manera muy negativa la práctica de sacar niños del aula para el trabajo con profesionales PIE.

» PIE se sigue relacionando con prácticas segregadoras por parte de todas las escuelas analizadas.

» No hay claridad de lo que pasa con PIE y los niños y niñas que son parte del programa en términos de que no hay claridad respecto de cuáles son los criterios para definir a PIE como un programa exitoso en el caso por caso.

\section{Conclusiones}

A través de este trabajo de re-conocimiento de las escuelas rurales en el país desde la perspectiva de la inclusión, es interesante ver cómo PIE es un programa que toma distintos colores y formas según la escuela. La apertura de él reflejada en el Decreto 170 podría tener un devenir distinto según la escuela. La experiencia de la escuela 4, con PIE y alto trabajo en aula, es un ejemplo de una transformación interesante de los planteamientos del proyecto, discutidos por la pequeña comunidad docente, puestos en marcha de manera coherente con un proyecto donde la inclusión se entiende como un proceso social donde lo colectivo tiene un rol importante y el diagnóstico ya no viene a cumplir un rol de gestión disciplinar del aula. Por otro lado, la práctica de articulación curricular y entre profesionales permite abordar el objetivo declarado por el programa respecto de favorecer el aprendizaje de todas y todos los estudiantes; esto coincide con otros estudios que muestran que es una práctica tradicionalmente escasa, pero que paulatinamente comienza a aumentar (Cornejo, 2017). Este trabajo coordinado sería el que se condice con el objetivo declarado por PIE en relación a favorecer la presencia de los estudiantes en la sala de clases (Mineduc, 2013). 
Sin embargo, la estructura y modelo de financiamiento de PIE promueven una mayor atención a lo individual que a lo grupal, es decir, las N.E.E se experimentan individualmente y por ende conllevan un diagnóstico individual disciplinante que implica la salida del aula para la atención de casos particulares. Esta articulación forma parte de una relación histórica, largamente establecida entre la pedagogía y la psicología (o disciplinas afines) lo que Ana Bahía Bock (2003) define como complicidad ideológica, que se traduce principalmente en la psicopatologización de los estudiantes y, por ende, en la responsabilización individual del fracaso escolar. Este discurso se encuentra latente en el programa de integración, así como también en el proceso de articulación que ocurre dentro de la mayoría de las escuelas.

PIE a la vez genera fuertes cuestionamientos de parte de algunos docentes, especialmente en aquellas escuelas donde el programa no existe. El diagnóstico es visto más como un impedimento que como una forma de inclusión. Si bien es necesario plantear que la evaluación de PIE es muy particular a los proyectos educativos, las culturas imperantes y, como hemos visto, la situación territorial, la inclusión que promete el programa es cuestionada en todos los casos y presentada como un modelo que se impone y que no está pensado para ser adaptado.

En cuanto a la gestión de los profesionales PIE, es importante señalar que estos no reciben preparación para desempeñarse en el contexto de la educación rural y rotan indistintamente entre escuelas rurales y urbanas. Esto da cuenta que la política educativa asume por defecto que existe un modelo hegemónico de educación correspondiente al urbano.

PIE parece ser una buena instancia de trabajo multidisciplinar (tal como otros estudios lo han demostrado), pero con efectos paradójicos en la inclusión. De hecho, las escuelas analizadas que no lo tienen no consideran necesaria su implementación por temor, justamente, a los procesos de psicopatologización y posible etiquetaje de los estudiantes por parte de profesionales "externos" a la cultura escolar de cada establecimiento.

Finalmente, nos parece urgente seguir investigando el posible tránsito entre niños y niñas que han pasado por programas PIE en escuelas urbanas (o rurales) a escuelas rurales con poca matrícula. La cultura más inclusiva de las escuelas rurales, junto con su necesidad de tener más matrícula, podrían generar un nuevo panorama, convirtiendo a los "niños PIE" en niños nómades del sistema escolar que vagan buscando refugio en escuelas que puedan integrar sus supuestas diferencias. Es extremadamente urgente analizar este problema con el ánimo de no poner sobre las escuelas rurales la solución a un problema no esperado, pero provocado por la propia política pública que pretende incluir. 


\section{Referencias}

Bahía Bock, A. (2003). Psicología de la educación: complicidad ideológica. En Psicología Escolar: teorías críticas (pp. 79-103). Bock, A.M.B.; Checcia, A. K. A.; Rebello de Souza, M. P., Casa do Psicólogo: Sao Paulo.

Balcázar, F. (2003). Investigación acción participativa (iap): Aspectos conceptuales y dificultades de implementación. Fundamentos en Humanidades, 4 (7-8), 59-77.

Booth, T. Ainscow, M., Black-Hawkins, K., Vaughan, M. y Shaw, L. (2000). Índice de Inclusión. Desarrollando el aprendizaje y la participación en las escuelas. Bristol: Centre for Studies on Inclusive Education (CSIE).

Carr, W. y Kemmis, S. (1988). Teoría crítica de la enseñanza, la investigación-acción en la formación del profesorado. Madrid: Ediciones Martínez Roca.

Centro de Innovación en Educación y Fundación Chile. (2013). Análisis de la Implementación de los Programas de Integración Escolar (PIE) en Establecimientos que han incorporado Estudiantes con Necesidades Educativas Especiales Transitorias (NEET). Documento Resumen del Estudio.

Corbin, J., \& Strauss, A. (2008). Basics of Qualitative Research: Techniques and Procedures for Developing Grounded Theory (3rd ed.). Thousand Oaks, CA: Sage.

Corvalán, J. (2006). Educación para la población rural en siete países de América Latina. Síntesis y análisis global de resultados por países. Revista Colombiana De Educación, (51). https://revistas.pedagogica.edu.co/ index.php/RCE/article/view/7684

Chile. Mineduc (2009). Criterios y orientaciones de flexibilización del curriculum. Para dar respuesta a la diversidad en los distintos niveles y modalidades de enseñanza. https://especial.mineduc.cl/wpcontent/ uploads/sites/31/2016/08/Criterios_Orientaciones_Flexibilizacion_ Curricular-2009.pdf

Chile. Mineduc (2017). Programa de Integración Escolar PIE. Manual de apoyo a la inclusión escolar en el marco de la Reforma educacional. https:/especial.mineduc.cl/wp-content/uploads/sites/31/2016/09/ Manual-PIE-Julio2017.pdf

Cornejo, C. (2017). Respuesta educativa en la atención a la diversidad desde la perspectiva de profesionales de apoyo. Revista Colombiana de Educación, (73), 77-96.

Elige Educar (2019). Radiografía de la educación rural en Chile. https:// eligeeducar.cl/radiografia-de-la-educacion-rural-en-chile 
Elliot, J. (2005). La investigación-acción en educación. Madrid: Ediciones Morata, S. L.

Íniguez, Lupicinio. (2004). Análisis del discurso. Manual para las ciencias sociales. Barcelona: Editorial UOC.

McEwan, P., y Carnoy, M. (2000). The effectiveness and efficiency of private schools in Chile's voucher system. Educational Evaluation and Policy Analysis, 22 (3), 213 - 239. https://doi. org/10.3102/01623737022003213

McEwan, P., Urquiola, M., Vegas, E., Fernandes, R., y Gallego, F. A. (2008). School choice, stratification, and information on school performance: Lessons from Chile. Economía, 8(2), 1- 42.

Ministerio de Educación. (2010, 21 de abril). Decreto con toma de razón $N^{\circ}$ 170. Fija normas para determinar los alumnos con necesidades educativas especiales que serán beneficiarios de las subvenciones para educación especial. Biblioteca Congreso Nacional.

Ministerio de educación. (2013). Orientaciones técnicas para Programas de Integración Escolar (PIE). División de Educación General, Unidad de Educación Especial. Santiago de Chile. https://especial.mineduc.cl/wp-content/uploads/sites/31/2016/09/Orientaciones-PIE-2013-3.pdf

Ministerio de Educación. (2015). Decreto n83. Aprueba criterios y orientaciones de adecuación curricular para estudiantes con necesidades educativas especiales de educación parvularia y educación básica. Biblioteca Congreso Nacional.

Ministerio de Educación. (2015, 8 de junio). Ley 20.845. De inclusión escolar que regula la admisión de los y las estudiantes, elimina el financiamiento compartido y prohíbe el lucro en establecimientos educacionales que reciben aportes del estado. Biblioteca Congreso Nacional.

Ministerio de Educación. (2016). Programa de Integración Escolar (PIE). Manual de apoyo a la inclusión escolar en el marco de la Reforma educacional. https://especial.mineduc.cl/wp-content/uploads/ sites/31/2016/09/Manual-PIE-Julio2017.pdf

Ministerio de Educación de Chile. (2009). Criterios y orientaciones de flexibilización del curriculum. Para dar respuesta a la diversidad en los distintos niveles y modalidades de enseñanza. https://especial. mineduc.cl/wp-content/uploads/sites/31/2016/08/Criterios_Orientaciones_Flexibilizacion_Curricular-2009.pdf

Ministerio de Educación (2019, 5 de agosto). Datos abiertos. http://datosabiertos.mineduc.cl/ 
Núñez, C. G., Peña, M., Cubillos, F. y Solorza, H. (2016). Estamos todos juntos: el cierre de la Escuela Rural desde la perspectiva de los niños. Educação e Pesquisa, 42(4), 953-967. http://dx.doi.org/10.1590/ s1517-9702201612152334

Núñez, C. G., Solís, C. y Soto, R. (2014). ¿Qué sucede en las comunidades cuando se cierra la escuela rural? Un análisis psicosocial de la política de cierre de las escuelas rurales en Chile. Revista Universitas Psychologica, 13(2), 615-625. https://revistas.javeriana.edu.co/ index.php/revPsycho/article/view/3602

Peña, M. (2013). Análisis crítico de discurso del decreto 170 de subvención diferenciada para necesidades educativas especiales: El diagnóstico como herramienta de gestión. Psicoperspectivas, 12(2), 9-103. https://www.psicoperspectivas.cl/index.php/psicoperspectivas/article/view/252

Rojas, T. y Armijo, M. (2016). Qué es la inclusión escolar: distintas perspectivas en debate. Cuaderno de Educación, 75, 1-12.

Silva Ríos, C. y Burgos Dávila, C. (2011). Tiempo mínimo-conocimiento suficiente: la cuasi-etnografía sociotécnica en psicología social. Psicoperspectivas, 10(2), 87-108.

Solís, C. y Núñez, C. G. (2014). El cierre de la escuela rural y la integración social de familias en escuelas urbanas: un estudio de caso en la zona sur de Chile. Revista Iberoamericana de Evaluación Educativa, 7(3), 57-69. https://dialnet.unirioja.es/descarga/articulo/5133423.pdf

Tamayo, M., Arrau, M., Sánchez, M., Besoaín, A. y Rebolledo, J. (2018). Programa de Integración Escolar en Chile: brechas y desafíos para la implementación de un programa de educación inclusiva. Revista Española de Discapacidad (REDIS), 6(1), 161-179. https://www.cedd. net/redis/index.php/redis/article/view/301

Trimano, L. (2015). Integración social y nueva ruralidad: ser ¿“hippie"? en el campo. Revista de Antropología Social, 24, 317-348.

Vásquez, F. S. (1994). Análisis de contenido categorial: el análisis temático. Unitat de Psicología Social. Universitat Autónoma de Barcelona. https://es.scribd.com/document/235557297/Analisis-de-Contenido-tematico-Felix-Vasquez-pdf

Vera-Bachmann, D. y Salvo, S. (2016). Perfiles de escuelas rurales exitosas: Una propuesta de la psicología educacional ante el cierre de escuelas. Universitas Psychologica, 15(2), 25-38. https://doi. org/10.11144/Javeriana.upsy15-2.perep 